\title{
On Spatial Scales and Lifetimes of SST Anomalies beneath a Diffusive Atmosphere
}

\author{
JoCHEM MAROTZKE \\ Center for Global Change Science, Department of Earth, Atmospheric, and Planetary Sciences, Massachusetts Institute of Technology, \\ Cambridge, Massachusetts \\ DAVID W. PIERCE \\ Climate Research Division, Scripps Institute of Oceanography, La Jolla, California
}

(Manuscript received 13 February 1996, in final form 31 May 1996)

\begin{abstract}
The authors identify spatial and temporal scales in a one-dimensional linear, diffusive atmospheric energy balance model coupled everywhere to a slab mixed layer of fixed depth. Mathematically, the model is identical to a heat conducting rod, which over its entire length both radiates and is in contact with a large but finite "reservoir." Three characteristic timescales mark, respectively, the atmosphere's adjustment to a sea surface temperature (SST) anomaly, the decay of a pointwise SST anomaly, and the radiative decay of a large-scale SST anomaly. The first and the third of these timescales are associated with diffusive length scales characterizing, respectively, the distance over which heat is diffused in the atmosphere before being lost to the ocean beneath, and the distance over which heat is diffused in the coupled system before being radiated to space. For spatial scales between the two diffusive lengths, the SST anomaly does not decay exponentially but with the square root of time; this regime has not previously been identified. Apparent discrepancies between published discussions of diffusive length scales are reconciled.
\end{abstract}

\section{Introduction}

We discuss what might appear to be a very simple problem: Given an isolated anomaly in mixed layer temperature, unaffected by interior oceanic dynamics but in contact with a one-dimensional atmosphere of fixed vertical structure and with purely diffusive horizontal transport, what characteristic spatial scales do subsequently emerge and what is the lifetime of this mixed layer temperature (or sea surface temperature, SST) anomaly? The question is motivated by the recent intense discussion in the literature about how SST boundary conditions in ocean general circulation models (GCMs) affect the stability of the thermohaline circulation, and about the intimate connection between atmospheric heat transports and equivalent surface heat flux formulations, as a function of SST anomalies (Zhang et al. 1993; Power and Kleeman 1994; Mikolajewicz and Maier-Reimer 1994; Rahmstorf and Willebrand 1995; Pierce et al. 1996; Chen and Ghil 1996). Conceptual models and the feedback processes involved in the interactions between atmospheric transports and the thermohaline circulation have been discussed by

Corresponding author address: Dr. Jochem Marotzke, Department of Earth, Atmospheric, and Planetary Science, Massachusetts Institute of Technology, Cambridge, MA 02139-4307.
Willebrand (1993), Marotzke (1994, 1996), Nakamura et al. (1994), Marotzke and Stone (1995), Saravanan and McWilliams (1995), and Rahmstorf et al. (1996).

The starting point for this paper is the concept, formulated (apparently independently) by Bretherton (1982), Frankignoul (1985), and Schopf (1985), that if interactions between SST anomalies and atmospheric dynamics obey linear laws, the damping of a small-scale anomaly should be strong, as expressed by local bulk formulas (Haney 1971). If the anomaly is large scale, the damping is weak, governed essentially by longwave radiation to space. The rationale is that on small scales atmospheric transports can efficiently transport heat horizontally and effect changes in SST outside the location of the original anomaly. On a global scale, only longwave radiation can act. Willebrand (1993) suggested an implementation of scale-selective SST damping into a GCM, which was subsequently done by Rahmstorf and Willebrand (1995).

The next conceptual question is against which length scale "small" and "large" are to be measured. Surprisingly, two very different answers were given by Marotzke (1994, M94 hereinafter) and Pierce et al. (1996, PKB hereinafter), although both papers employed linear diffusive energy balance models (the latter a considerably more sophisticated one). A diffusive length scale was derived in both cases, but M94 chose 
as the characteristic timescale the local atmospheric adjustment timescale, essentially as given through a Haney relaxation coefficient, whereas PKB selected the time it takes longwave radiation to eliminate a local anomaly in total, atmosphere plus ocean, heat content, which naturally does not invoke the air-sea coupling coefficient for heat transfer. The resulting length scales differ by almost an order of magnitude.

The discrepancy between two different scale estimates of such an important theoretical quantity motivates this investigation of the initial value problem of a one-dimensional diffusive rod, which is slowly leaking heat to one side ("space") and which is tightly coupled to a large but finite "reservoir" on the other side. M94 solved this problem if the mixed layer was truly a reservoir; that is, its temperature was assumed fixed. A very similar model was analyzed by Power et al. (1995). However, the mixed layer's heat capacity is large but finite, compared to the atmosphere's, so here we take the significant additional step of computing how the SST anomaly evolves after the atmosphere has equilibrated (section 2). Schopf (1985) analyzed a model similar to ours and explicitly demonstrated the scale sensitivity of SST damping timescales. This manuscript goes beyond Schopf's (1985) work in that the conditions for the validity of various limiting cases are formulated quantitatively and in that the various stages of SST damping are analyzed explicitly. Moreover, we are able to reconcile the seemingly contradictory results of M94 and PKB. A brief discussion follows in section 3 .

\section{Model solutions}

A single-level atmospheric model is coupled to a slab ocean of uniform thickness, both of which have infinite horizontal extent in one dimension only. Heat is transported horizontally by diffusion in the atmosphere, and heat is lost from the system via longwave radiation to space. Horizontal heat transport in the ocean is neglected. The atmosphere and ocean exchange heat at a rate that is proportional to the temperature difference between them, and the evolution of the ocean model temperature is totally determined by this exchange.

The model is defined through the heat conservation equations for atmosphere and ocean,

$$
\begin{aligned}
& c_{A} \partial_{t} T_{A}=-B T_{A}+c_{A} D \partial_{y y} T_{A}+\lambda\left(T_{O}-T_{A}\right), \\
& c_{O} \partial_{t} T_{O}=-\lambda\left(T_{O}-T_{A}\right),
\end{aligned}
$$

where $c_{A}=10^{7} \mathrm{~W} \mathrm{~s} \mathrm{~m}^{-2}$ and $c_{O}=2 \times 10^{8} \mathrm{~W} \mathrm{~s} \mathrm{~m}^{-2}$ are the heat capacities per unit area of atmosphere and mixed layer, respectively; $B=2 \mathrm{~W} \mathrm{~m}^{-2} \mathrm{~K}^{-1}$ is the longwave radiation coefficient; $D=2.5 \times 10^{6} \mathrm{~m}^{2} \mathrm{~s}^{-1}$ the atmospheric diffusivity (e.g., Stone and Miller 1980); and $\lambda$ the air-sea exchange coefficient, which is as yet unspecified but always much larger than B. All temperatures are deviations from some equilibrium, and a purely one-dimensional model is considered (the two- dimensional case with radial symmetry is likely to give qualitatively similar results). The terms on the righthand side of Eq. (1) are readily identified as longwave radiation to space, horizontal diffusion, and ocean-toatmosphere heat flux. Initial conditions are prescribed; for simplicity, the model domain extends to infinity. The atmosphere is thus equivalent to an infinite rod, with radiative loss to one side, and the ocean a large but finite reservoir on the other without any interior heat transport process.

Although the physical problem appears very simple, the coupled equations (1) and (2) are surprisingly complex, and we have been unable to either calculate the exact solution or locate it in any of the standard references on diffusion (e.g., Crank 1975; Carslaw and Jaeger 1986). It is likely that the general solution, if it can be given in closed form, will be quite unyielding to ready interpretation, so we have chosen instead to consider a suite of approximations to (1) and (2), and solve the approximated equations. Overall, this has proven more illuminating than performing the asymptotics on a more general solution.

Equations (1) and (2) can be rewritten without approximation

$$
\begin{aligned}
\left(1+\frac{c_{A}}{\lambda} \partial_{t}\right) T_{A}-\frac{c_{A}}{\lambda} D \partial_{y y} T_{A}+\frac{B}{\lambda} T_{A} & =T_{O} \\
\left(1+\frac{c_{O}}{\lambda} \partial_{t}\right) T_{O} & =T_{A} .
\end{aligned}
$$

Two timescales, $\tau_{1} \equiv c_{A} / \lambda$ and $\tau_{2} \equiv c_{O} / \lambda$, are immediately identified, characterizing the adjustment of atmospheric and oceanic temperatures to a restoring surface heat flux. A third timescale, $\tau_{3} \equiv c_{o} / B$, characterizes the adjustment of the coupled system through longwave radiation to space. For $\lambda=50 \mathrm{~W} \mathrm{~m}^{-2} \mathrm{~K}^{-1}$, the timescales are, in this order, 2 days, 50 days, and 3 years. We will introduce approximations to Eqs. (3) and (4) primarily by considering timescales long or short compared to $\tau_{1}, \tau_{2}$, or $\tau_{3}$. The assumptions about timescales will be stated at the beginning of each subsection; in addition it will be used throughout that $B \ll \lambda, c_{A} \ll$ $c_{O}$ (hence, $\tau_{1} \ll \tau_{2} \ll \tau_{3}$ ), and $O\left(T_{O}\right) \geq O\left(T_{A}\right)$. The timescale of interest will be denoted $\tau$.

\section{a. Timescales much shorter than atmospheric adjustment timescale, $\boldsymbol{\tau} \ll \boldsymbol{\tau}_{l}$}

We can write, symbolically,

$$
\frac{c_{O}}{\lambda} \partial_{t} \gg \frac{c_{A}}{\lambda} \partial_{t} \gg 1,
$$

so we only look at processes that occur faster than over two days. (Clearly, no energy balance model of the atmosphere constitutes a meaningful representation of the real world on these timescales.) Assuming that the initial 
perturbations in $T_{A}$ and $T_{O}$ are of the same order of magnitude, (3) and (4) simplify to

$$
\begin{aligned}
\partial_{t} T_{A}-D \partial_{y y} T_{A} & =0, \\
\partial_{t} T_{O} & =0 .
\end{aligned}
$$

Ocean and atmosphere are decoupled, and an initial anomaly in the atmosphere is dispersed by pure diffusion. The solution is given as a special case of Eq. (11) below.

\section{b. Timescales comparable to atmospheric adjustment timescale, $\boldsymbol{\tau} \sim \boldsymbol{\tau}_{l}$}

We now have

$$
\frac{c_{O}}{\lambda} \partial_{t} \gg \frac{c_{A}}{\lambda} \partial_{t} \sim 1,
$$

and we obtain for $T_{A}$ a diffusion equation with forcing and "radiation,"

$$
\begin{aligned}
\partial_{t} T_{A}-D \partial_{y y} T_{A}+\frac{\lambda}{c_{A}} T_{A} & =\frac{\lambda}{c_{A}} T_{O}, \\
c_{O} \partial_{t} T_{O} & =0 .
\end{aligned}
$$

In this limit, $T_{O}$ can be considered an externally prescribed forcing that is "switched on" at time $t=0$. The solution for $T_{A}$ is (Morse and Feshbach 1953, p. 121)

$$
\begin{aligned}
T_{A}(y, t)= & \frac{\lambda}{c_{A}} \int_{0}^{t} \frac{\exp \left[-\frac{\lambda}{c_{A}}\left(t-t^{\prime}\right)\right]}{2 \sqrt{\pi D\left(t-t^{\prime}\right)}} d t^{\prime} \\
& \cdot \int_{-\infty}^{+\infty} T_{O}\left(y^{\prime}, t^{\prime}\right) \exp \left[-\frac{\left(y-y^{\prime}\right)^{2}}{4 D\left(t-t^{\prime}\right)}\right] d y^{\prime} .
\end{aligned}
$$

This solution is readily understood if $T_{o}$ is a $\delta$ function in space $T_{o}\left(y^{\prime}, t^{\prime}\right)=\hat{T}_{o} \delta\left(y^{\prime}-y_{0}\right)$, for $t^{\prime}>0$, which leads to

$$
\begin{aligned}
T_{A}(y, t)= & \frac{\lambda}{c_{A}} \int_{0}^{t} \frac{\hat{T}_{O}}{2 \sqrt{\pi D\left(t-t^{\prime}\right)}} \\
& \cdot \exp \left[-\frac{\lambda}{c_{A}}\left(t-t^{\prime}\right)-\frac{\left(y-y_{0}\right)^{2}}{4 D\left(t-t^{\prime}\right)}\right] d t^{\prime} .
\end{aligned}
$$

In response to a localized stepwise increase in $T_{O}$, the atmosphere is heated above the SST anomaly (governed by the factor $\left.\lambda / c_{A} \equiv 1 / \tau_{1}\right)$. The atmospheric temperature anomaly is then diffused outward and lost through exchange with the ocean. At every instant, the ocean acts as a heat source at $y=y_{0}$.

\section{c. Timescales between atmospheric and oceanic adjustment timescales, $\boldsymbol{\tau}_{1} \ll \boldsymbol{\tau} \ll \boldsymbol{\tau}_{2}$}

Now,

$$
\frac{c_{O}}{\lambda} \gg 1 \gg \frac{c_{A}}{\lambda} \partial_{t},
$$

and we consider the diagnostic limit of the forced diffusion equation for the atmosphere, that is, the time rate of change of heat storage in the atmosphere is neglected, so that the atmosphere is in energy balance. We obtain

$$
\partial_{y y} T_{A}-\frac{\lambda}{D c_{A}} T_{A}=-\frac{\lambda}{D c_{A}} T_{O},
$$

with ocean temperature still unchanged from its initial state. Essentially, Eq. (14) is the one analyzed by M94 (who included the longwave radiation term, which is trivial on these timescales). The solution is

$$
T_{A}(y)=\frac{1}{2 L_{1}} \int_{-\infty}^{+\infty} T_{O}\left(y^{\prime}\right) e^{-\left(\mid y-y^{\prime}\right) / L_{1}} d y^{\prime},
$$

where

$$
L_{1} \equiv\left(D \tau_{1}\right)^{1 / 2} \equiv\left(\frac{D c_{A}}{\lambda}\right)^{1 / 2}
$$

is the diffusive length scale derived by M94, characterizing the distance over which heat emanating from an SST anomaly is transported in the atmosphere before being returned to the ocean. For the parameters chosen here, $L_{1}$ is about $700 \mathrm{~km}$. A Lagrangian interpretation states that a column of air, heated above the anomaly, flows over the colder ambient ocean and gets cooled again, with the $e$-folding time given by the atmospheric adjustment timescale. If the SST anomaly is spatially constant in an interval $L_{0}$,

$$
T_{o}(y)= \begin{cases}\hat{T}_{o}, & |y| \leq L_{0} / 2 \\ 0, & |y|>L_{0} / 2,\end{cases}
$$

the atmospheric temperature is readily determined as

$$
T_{A}(y)= \begin{cases}\hat{T}_{O}\left(1-e^{-L_{0} / 2 L_{1}} \cosh \frac{y}{L_{1}}\right), & |y| \leq L_{0} / 2 \\ \hat{T}_{O} \sinh \frac{L_{0}}{2 L_{1}} e^{-y / L_{1}}, & |y|>L_{0} / 2 .\end{cases}
$$

For a large-scale SST anomaly, $L_{0} \gg L_{1}$, Eq. (18) gives approximately that $T_{A} \approx \hat{T}_{O}$ above the anomaly except in a transition region of width $L_{1}$ around $y=$ $\pm L_{0} / 2$, where $T_{A}$ drops to zero. For a small-scale SST anomaly, $L_{0} \ll L_{1}$, one obtains that

$$
T_{A}(y) \cong \begin{cases}\hat{T}_{o} \frac{L_{0}}{2 L_{1}}, & |y| \leq L_{0} / 2 \\ \hat{T}_{o} \frac{L_{0}}{2 L_{1}} e^{-y / L_{1}}, & |y|>L_{0} / 2,\end{cases}
$$

meaning that if $\epsilon \equiv L_{0} / L_{1}, T_{A}=O\left(\epsilon \hat{T}_{O}\right)$ everywhere within the diffusive transport scale $\mathrm{L}_{1}$ of the anomaly. As a consequence, the atmosphere-minus-ocean temperature difference is $O\left(\epsilon \hat{T}_{o}\right)$ outside the SST anomaly, where $T_{O}=0$, but it is negative and $O\left(T_{O}\right)$ right above the anomaly. Hence, the surface heat flux is vigorous 
above the anomaly, and of opposite sign and weaker by a factor of $\epsilon$ outside. The spatial integral of surface heat flux is zero within the approximation made here. That the atmospheric temperature anomaly is "small" above a small-scale SST anomaly was previously found by Schopf (1985), who noted that, hence, the assumption of an unchanging atmospheric temperature, which underlies Haney's (1971) parameterization, is well justified for small-scale SST anomalies.

\section{d. Timescales comparable to oceanic adjustment timescale, $\boldsymbol{\tau} \sim \boldsymbol{\tau}_{2}$}

We now move into the regime where the SST responds to the change in heat flux, while the atmosphere goes through a sequence of equilibrium responses to each new SST field. The response of the SST depends strongly on the size of the anomaly, as illustrated by Eqs. (18) and (19). For a large-scale anomaly $\left(L_{0} \gg\right.$ $\left.L_{1}\right)$, atmospheric and oceanic temperatures are very close; the discussion is deferred to the next subsection. For a very small-scale anomaly, the air-sea temperature differential above the anomaly is almost as great as the anomaly itself; hence, SST decays exponentially on a timescale given by $c_{O} / \lambda$. The heat lost by the ocean is transported by the atmosphere over distance $L_{1}$ and returned to the ocean. Thus, $L_{1}$ also marks the extent of the SST anomaly when the exponential cooling stops.

\section{e. Timescales between oceanic adjustment timescale and oceanic radiative damping timescale, $\tau_{2} \ll \tau$ $\leq \boldsymbol{\tau}_{3}$}

For the subsequent development, we can no longer ignore the contribution of the longwave radiation. For example, Eq. (1) indicates that if the air-sea temperature difference is less than $O\left(T_{A} \times B / \lambda\right)$, as is the case above a large-scale SST anomaly, the surface heat flux does not dominate the longwave flux.

We now take the steady-state limit of the atmospheric heat conservation equation (1) and eliminate $T_{\mathrm{A}}$ by inserting the oceanic heat conservation equation (4), which yields

$$
c_{O} \partial_{t} T_{O}+\left(1+\frac{c_{O}}{\lambda} \partial_{t}\right)\left(B-c_{A} D \partial_{y y}\right) T_{O}=0 .
$$

Under the assumption that only timescales longer than the oceanic adjustment timescale are considered,

$$
\frac{c_{O}}{\lambda} \partial_{t} \ll 1,
$$

the only term involving $\lambda$ drops out, and Eq. (20) reduces to

$$
\partial_{t} T_{o}-\frac{c_{A}}{c_{o}} D \partial_{y y} T_{o}+\frac{B}{c_{0}} T_{o}=0 .
$$

Equation (22) was obtained heuristically by PKB who considered the evolution of total heat content [the sum of Eqs. (1) and (2)], neglected atmospheric heat content, and equated $T_{A}$ with $T_{O}$. We see that the last step is justified only for large enough timescales which, by the considerations of the previous subsection, implies spatial scales larger than $L_{1}$. The derivation of PKB emphasizes that (22) is an equation for the coupled system. Here, we point out that it is a diffusion equation with a horizontal diffusivity that is reduced by a factor of $c_{A} /$ $c_{O}$ compared to the atmospheric diffusivity. The solution to arbitrary initial conditions $T_{o}\left(y^{\prime}, 0\right)$ is

$$
\begin{aligned}
T_{o}(y, t)= & \frac{\exp \left(-\frac{B}{c_{O}} t\right)}{2 \sqrt{\pi \frac{c_{A}}{c_{O}} D t}} \\
& \cdot \int_{-\infty}^{+\infty} T_{O}\left(y^{\prime}, 0\right) \exp \left[-\frac{\left(y-y^{\prime}\right)^{2}}{4 \frac{c_{A}}{c_{O}} D t}\right] d y^{\prime} .
\end{aligned}
$$

The initial anomaly "freely" diffuses out until the time $\tau_{3} \equiv c_{O} / B$ has passed and longwave radiation begins to reduce the total heat content anomaly significantly, limiting the distance over which the anomaly is spread. The associated length scale is the one derived by PKB and given by

$$
L_{3} \equiv\left(D \tau_{3}\right)^{1 / 2} \equiv\left(\frac{D c_{O}}{B}\right)^{1 / 2} .
$$

For the parameters chosen here, $\mathrm{L}_{3}$ is around $4000 \mathrm{~km}$. The Green's function solution to Eq. (23) indicates that the peak value of the anomaly decays as $t^{1 / 2}$, for times much shorter than $\tau_{3}$.

\section{f. Timescales much longer than oceanic radiative timescale, $\boldsymbol{\tau} \gg \boldsymbol{\tau}_{3}$}

Now,

$$
\frac{c_{O}}{B} \partial_{t} \ll 1
$$

and we can neglect the first time derivative in Eq. (20) as well. The resulting equation has only the trivial solution for homogeneous boundary conditions, meaning that the SST anomaly has completely vanished through longwave radiation to space.

\section{Discussion}

The fate of a pointwise SST anomaly that suddenly comes into existence underneath a diffusive atmosphere can be summarized as follows. (Without loss of generality, the anomaly is assumed positive.) First, the at- 

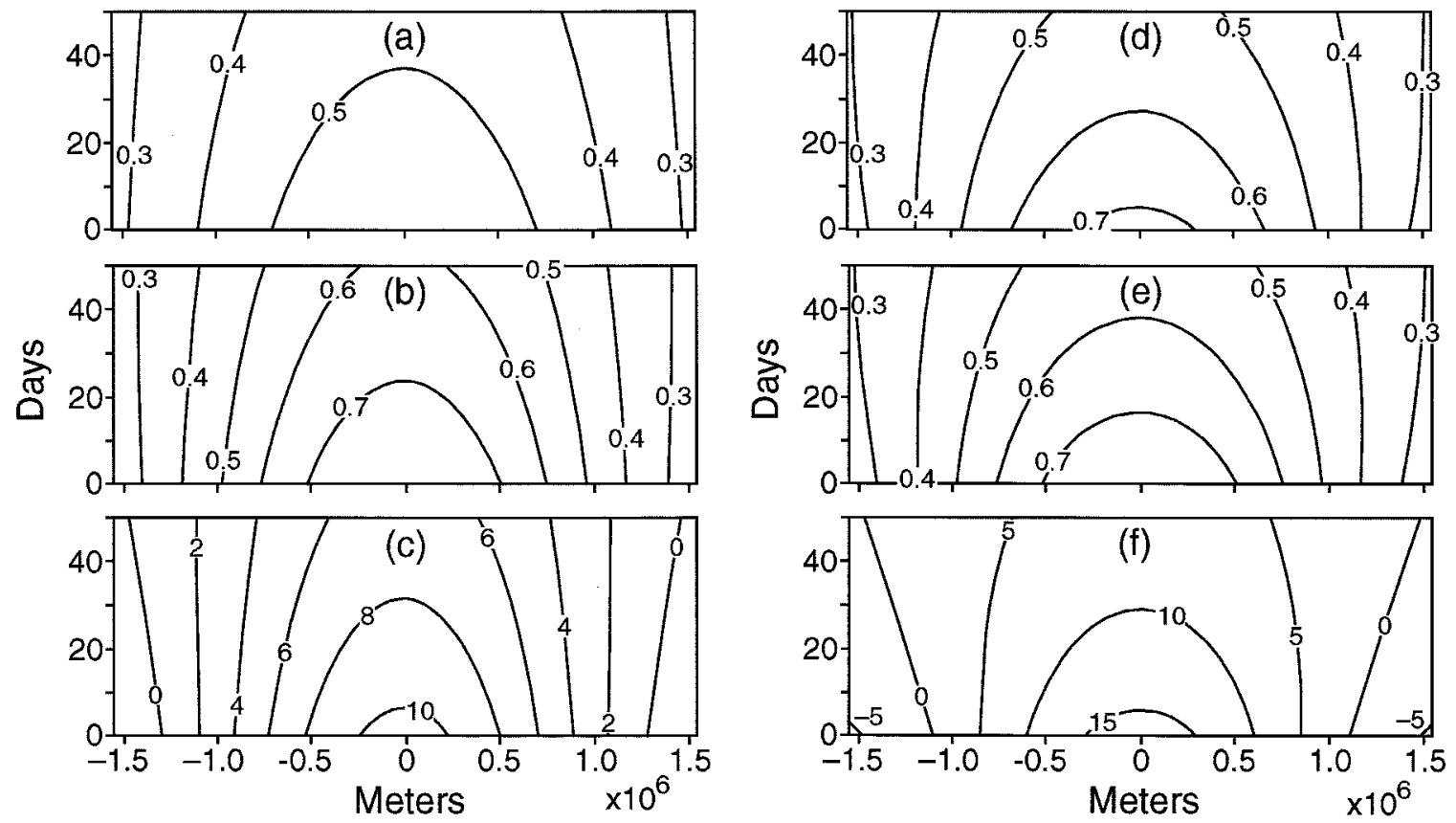

FIG. 1. Numerical solution of the coupled model (diagnostic atmosphere), as a function of time and latitude (the domain is not shown completely). The initial anomaly is a Gaussian bump with half-width $1000 \mathrm{~km}$; the peak initial value is chosen such that total initial heat content is equal to that of a uniform anomaly of $1^{\circ} \mathrm{C}$, of size $2000 \mathrm{~km}$. (a) Atmospheric temperature, $\lambda=50 \mathrm{~W}$ m ${ }^{-2} \mathrm{~K}^{-1}$. (b) Oceanic temperature, $\lambda=50 \mathrm{~W} \mathrm{~m}^{-2} \mathrm{~K}^{-1}$. (c) Ocean-to-atmosphere heat flux, $\lambda=50 \mathrm{~W} \mathrm{~m}^{-2} \mathrm{~K}^{-1}$. (d) Atmospheric temperature, $\lambda=250 \mathrm{~W}$ m ${ }^{-2}$ $\mathrm{K}^{-1}$. (e) Oceanic temperature, $\lambda=250 \mathrm{~W} \mathrm{~m}^{-2} \mathrm{~K}^{-1}$. (f) Ocean-to-atmosphere heat flux, $\lambda=250 \mathrm{~W} \mathrm{~m}^{-2} \mathrm{~K}^{-1}$.

mosphere above the anomaly is heated on a timescale $\tau_{1} \equiv c_{A} / \lambda \sim 2$ days, where $c_{A}$ is the atmospheric heat capacity and $\lambda$ the air-sea heat exchange coefficient. This localized atmospheric temperature anomaly is then diffused out, governed by atmospheric diffusivity $D$. On timescales over which the SST is changed by the heat flux anomalies, the atmosphere has equilibrated. Heat lost by the ocean above the original SST anomaly is transported over a distance $L_{1} \sim 700 \mathrm{~km}$ given by the atmospheric adjustment timescale and the atmospheric diffusivity [Eq. (16) and Marotzke 1994] and returned to the ocean. Air-sea temperature differences are of the order of the original SST anomaly above the anomaly, and smaller outside by a factor of anomaly extent divided by $L_{1}$. Consequently, the SST anomaly decays exponentially on a timescale $\tau_{2} \equiv c_{O} / \lambda \sim 50$ days, where $c_{O}$ is the mixed layer heat capacity. At the end of the exponential decay phase, the anomaly has a size of the order of $L_{1}$.

If the SST anomaly has a spatial scale much larger than $L_{1}$, either right from the start or because it has been expanded by atmospheric diffusion, the ocean-atmosphere temperature difference virtually vanishes above the anomaly. The anomaly is spread diffusively, but now with diffusivity $D c_{A} / c_{O}$. The diffusion is limited by longwave radiation to space, which occurs on a timescale $\tau_{3} \equiv c_{O} / B \sim 3$ years, where $B$ is the longwave radiation coefficient. The distance $L_{3} \sim 4000 \mathrm{~km}$ over which the SST anomaly can be transported in time $\tau_{3}$, is given in
Eq. (24) and Pierce et al. (1996). We are thus able to reconcile the apparent contradiction between the diffusive length scales derived by Marotzke (1994) and Pierce et al. (1996). Scale $L_{1}$ defines the distance over which atmospheric diffusion can transport heat away from an SST anomaly before returning it to the ocean. Scale $\mathrm{L}_{3}$ defines how far the (weaker) diffusion of the coupled anomaly traverses before longwave radiation enters. It follows that $L_{1}$ and $L_{3}$ divide spatial scales into three regimes, each with very different decay. SST anomalies much smaller than $\mathrm{L}_{1}$ decay exponentially on a timescale given essentially by the local bulk coefficient $\lambda$ (Haney 1971). SST anomalies much larger than $L_{3}$ decay exponentially on a timescale determined by the longwave radiation coefficient $B$. The range of intermediate spatial scales, between $L_{1}$ and $L_{3}$ and well separated from both, defines a dynamical regime ("free diffusion" of the anomaly in total heat content) that has hitherto not been identified. The peak value of the anomaly does not decay exponentially but with the square root of time.

A numerical solution of Eqs. (1) and (2) is displayed in Fig. 1. Equation (1) is solved in the diagnostic limit; Fig. 1 shows atmospheric and oceanic temperatures and surface heat flux, as a function of time and space (the domain is not shown completely). The air-sea exchange coefficient $\lambda$ is $50 \mathrm{~W} \mathrm{~m}^{-2} \mathrm{~K}^{-1}$ in Figs. $1 \mathrm{a}-\mathrm{c}$ and 250 $\mathrm{W} \mathrm{m}^{-2} \mathrm{~K}^{-1}$ in Figs. 1d-f. The initial anomaly is a Gaussian bump with half-width $1000 \mathrm{~km}$; the peak initial value 


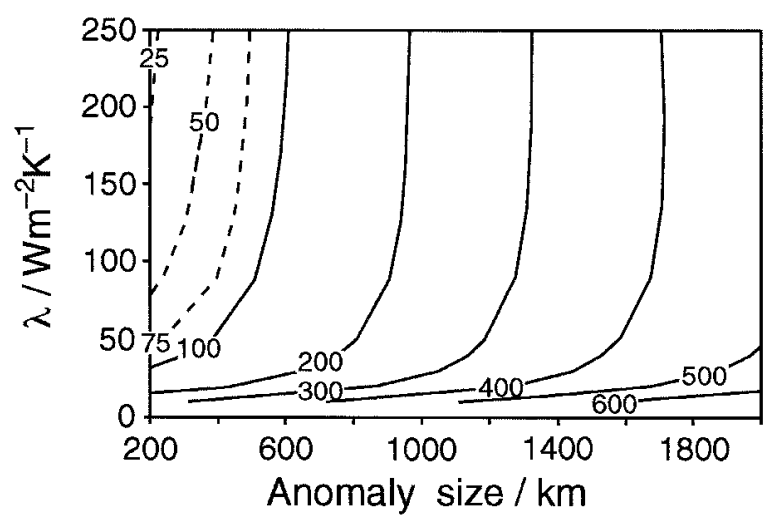

FIG. 2. SST lifetime in days as a function of $\lambda$ and anomaly size (Gaussian half-width). Lifetime is defined such that at the center of the anomaly, SST has dropped to $e^{-1}$ of its initial value.

is chosen such that total initial heat content is equal to that of a uniform anomaly of $1^{\circ} \mathrm{C}$ over $2000 \mathrm{~km}$. Both ocean and atmospheric temperatures decay more rapidly when $\lambda$ is large; consistently, ocean heat loss is more vigorous initially but is quickly reduced. Overall, Fig. 1 demonstrates that although the initial SST anomaly is rather large scale, its evolution still depends on $\lambda$. This sensitivity is even more pronounced in the phase space diagram, Fig. 2, showing after how many days the SST at the center of the anomaly has dropped to $e^{-1}$ of its initial value, as a function of $\lambda$ and anomaly size. For SST anomalies several $100 \mathrm{~km}$ wide, lifetimes change with $\lambda$ when $\lambda$ is as high as $250 \mathrm{~W} \mathrm{~m}^{-2} \mathrm{~K}^{-1}$. But even for anomalies with a Gaussian half-width of $1500 \mathrm{~km}$, the sensitivity of lifetime to $\lambda$ tapers off only if $\lambda$ is greater than $100 \mathrm{~W} \mathrm{~m}^{-2} \mathrm{~K}^{-1}$, that is, outside the range that is physically sensible. This dependence is consistent with Chen and Ghil's (1996) results from a coupled ocean GCM-energy balance model, where the existence of interdecadal variability of the thermohaline circulation depended on both the horizontal atmospheric diffusivity and on the local air-sea exchange coefficient.

Rahmstorf and Willebrand (1995) and Capotondi and Saravanan (1996) likewise employed a linear energy balance model coupled to ocean models but replaced atmospheric temperature by oceanic temperature in the expressions for longwave radiation and atmospheric diffusion. This is equivalent to letting $\lambda$ go to infinity and hence $L_{1}$ to zero; a preferable physical interpretation avoiding the singularity is that the spatial scale of the SST anomaly is assumed always to be much larger than $L_{1}$. Since $L_{1}$ is about $700 \mathrm{~km}$ for a $\lambda$ given by standard bulk formulas $\left(50 \mathrm{~W} \mathrm{~m}^{-2} \mathrm{~K}^{-1}\right)$, this latter condition is not always fulfilled even for coarse-resolution models (gridsize about $4^{\circ}$ ). Hence, the assumption of Rahmstorf and Willebrand (1995, p. 790) that "on the space and time scales important for ocean climate experiments the air temperature is dominated by the local coupling to the ocean temperature and not by atmospheric heat transport" is not always justified [see Eq. (19) and
Schopf 1985]. Conversely, the choice of $\lambda$ influences the lifetimes of all but the very largest-scale SST anomalies in coarse-resolution models, as demonstrated in Figs. 1 and 2 and indirectly by Chen and Ghil (1996). Overall, we conclude that solving an explicit energy balance model is preferable to the approximation of assuming that the atmospheric transport length scale is much smaller than the gridsize.

It seems appropriate to conclude with a reminder of what processes important to ocean-atmosphere interaction are not described by this model. Effects of SST on mixed layer depth and ocean currents are neglected, besides making the unrealistic assumption of diffusive atmospheric heat transport. Atmospheric circulation is important in inducing SST anomalies (e.g., Frankignoul 1985; Cayan 1992; Deser and Blackmon 1993; Kushnir 1994), as are changes in ocean heat transport. Here, we only look at the time history of an SST anomaly after is has been created, irrespective of its origin. However, it seems possible to combine the insights gained here with a simple model for the creation of SST anomalies, perhaps along the lines demonstrated by Frankignoul (1985). Most directly, the analysis presented here serves the understanding of ocean GCMs coupled to atmospheric energy balance models, a configuration that has become increasingly popular lately.

Acknowledgments. We wish to thank an anonymous reviewer for constructive comments and for pointing out the Schopf (1985) reference. J. M. was partially supported by the Tokyo Electric Power Company through the TEPCO/MIT Environmental Research Program, and to 30 percent $(\$ 3,000)$ jointly by the Northeast Regional Center of the National Institute for Global Environmental Change and by the Program for Computer Hardware, Applied Mathematics, and Model Physics (both with funding from the U.S. Department of Energy).

\section{REFERENCES}

Bretherton, F. P., 1982: Ocean climate modeling. Progress in Oceanography, Vol. 11, Pergamon, 93-129.

Capotondi, A., and R. Saravanan, 1996: Sensitivity of the thermohaline circulation to surface buoyancy forcing in a two-dimensional ocean model. J. Phys. Oceanogr., 26, 1561-1578.

Carslaw, H. S., and J. C. Jaeger, 1986: Conduction of Heat in Solids. $2 \mathrm{~d}$ ed. Oxford University Press, $510 \mathrm{pp}$.

Cayan, D. R., 1992: Latent and sensible heat flux anomalies over the northern oceans: The connection to monthly atmospheric circulation. J. Climate, 5, 354-369.

Chen, F., and M. Ghil, 1996: Interdecadal variability in a hybrid coupled ocean-atmosphere model. J. Phys. Oceanogr., 26, $1039-1058$.

Crank, J., 1975. The Mathematics of Diffusion. 2d ed. Oxford University Press, $414 \mathrm{pp}$.

Deser, C., and M. L. Blackmon, 1993: Surface climate variations over the North Atlantic Ocean during winter: 1900-1989. J. Climate, 6, 1743-1753.

Frankignoul, C., 1985: Sea surface temperature anomalies, planetary waves, and air-sea feedback in the middle latitudes. Rev. Geophys., 23, 357-390. 
Haney, R. L. 1971: Surface thermal boundary condition for ocean circulation models. J. Phys. Oceanogr., 1, 241-248.

Kushnir, Y., 1994: Interdecadal variations in North Atlantic sea surface temperature and associated atmospheric conditions. J. Climate, 7, 141-157.

Marotzke, J., 1994: Ocean models in climate problems. Ocean Processes in Climate Dynamics: Global and Mediterranean Examples, P. Malanotte-Rizzoli and A.R. Robinson, Eds., Kluwer, 79-109.

- 1996: Analysis of thermohaline feedbacks. Decadal Climate Variability: Dynamics and Predictability. D.L.T. Anderson and J. Willebrand, Eds., Springer-Verlag, 333-378.

- , and P. H. Stone, 1995: Atmospheric transports, the thermohaline circulation, and flux adjustments in a simple coupled model. $J$. Phys. Oceanogr., 25, 1350-1364.

Mikolajewicz, U., and E. Maier-Reimer, 1994: Mixed boundary conditions in ocean general circulation models and their influence on the stability of the model's conveyor belt. J. Geophys. Res. 99, 22 633-22 644.

Morse, P. M. and H. Feshbach, 1953: Methods of Theoretical Physics. Parts 1 and 2, McGraw-Hill, 1978 pp.

Nakamura, M., P. H. Stone, and J. Marotzke, 1994: Destabilization of the thermohaline circulation by atmospheric eddy transports. J. Climate, 7, 1870-1882.

Pierce, D. W., K.-Y. Kim, and T. P. Barnett, 1996: Variability of the thermohaline circulation in an ocean general circulation mode coupled to an atmospheric energy balance model. J. Phys. Oceanogr., 26, 725-738.
Power, S. B., and R. Kleeman, 1994: Surface heat flux parameterization and the response of ocean general circulation models to high latitude freshening. Tellus, 46A, 86-95.

,-- R. A. Colman, and B. J. McAvaney, 1995: Modeling the surface heat flux response to long-lived SST anomalies in the North Atlantic. J. Climate, 8, 2161-2180.

Rahmstorf, S., and J. Willebrand, 1995: The role of temperature feedback in stabilizing the thermohaline circulation. J. Phys. Oceanogr., 25, 787-805.

- J. Marotzke, and J. Willebrand, 1996: Stability of the thermohaline circulation. The Warm Water Sphere of the North Atlantic Ocean, W. Krauss, Ed., Borntraeger, 129-157.

Saravanan, R., and J. C. McWilliams, 1995: Multiple equilibria, natural variability, and climate transitions in an idealized oceanatmosphere model. J. Climate, 8, 2296-2323.

Schopf, P. S., 1985: Modeling tropical sea-surface temperature: Implication of various atmospheric responses. Coupled Ocean-Atmosphere Models, J. C. J. Nihoul, Ed., Elsevier, 727-734.

Stone, P. H., and D. A. Miller, 1980: Empirical relations between seasonal changes in meridional temperature gradients and meridional fluxes of heat. J. Atmos. Sci., 37, 1708-1721.

Willebrand, J., 1993: Forcing the ocean with heat and freshwater fluxes. Energy and Water Cycles in the Climate System, E. Raschke, Ed., Springer-Verlag, 215-233.

Zhang, S., R. J. Greatbatch, and C. A. Lin, 1993: A reexamination of the polar halocline catastrophe and implications for coupled ocean-atmosphere modeling. J. Phys. Oceanogr., 23, 287-299. 